HRJ

V.2 n.11 (2021)

Recebido: $19 / 03 / 2021$

Aceito: 20/04/2021

\title{
Sífilis Congênita: atualização no manejo clínico no Distrito Federal
}

\author{
Ingrid de Sousa Veras ${ }^{1}$ \\ Mariana Viana Almeida ${ }^{2}$ \\ ${ }^{1}$ Enfermeira Residente do Programa Uniprofissional em Enfermagem em Obstetrícia da ESCS/FEPECS/SES- \\ DF \\ 2 Enfermeira Obstetra. Tutora do Programa Uniprofissional em Enfermagem em Obstetrícia da \\ ESCS/FEPECS/SES-DF
}

\section{RESUMO}

Devido ao aumento no número de casos de sífilis em gestante e juntamente com o crescimento da Sífilis Congênita (SC), houve a necessidade de criar um novo protocolo pelo Ministério da Saúde para prevenção da transmissão vertical da sífilis. Com isso, a Secretaria de Estado de Saúde do DF (SES-DF) emitiu a Nota Técnica N. ${ }^{\circ}$ 9/20 publicada em julho de 2020 para o manejo dos casos de SC no DF, baseada no protocolo atualizado. Este estudo tem como objetivo discutir sobre as novas recomendações para os profissionais da Atenção Primária em Saúde (APS) e maternidades do DF, relacionando-o com o caso. Trata-se de um relato de caso com recém-nascido exposto à sífilis em um hospital público do DF no setor do Alojamento Conjunto (ALCON) realizado no mês de agosto de 2020 durante a atividade prática obrigatória da residência uniprofissional em Enfermagem Obstétrica. Contudo, observou-se a importância de seguir as recomendações para minimizar danos e intervenções desnecessárias para a família e recém-nascido.

Palavras-chave: Sífilis Congênita. Treponema pallidum. Infecções sexualmente transmissíveis.

\section{Congenital Syphilis: updating clinical management in the Federal District}

\begin{abstract}
Due to the increase in the number of syphilis cases in pregnant women and along with the growth of Congenital Syphilis (SC), there was a need to create a new protocol by the Ministry of Health to prevent vertical transmission of syphilis. As a result, the State Department of Health of the DF (SES-DF) issued Technical Note No. 9/20 published in July 2020 for the management of SC cases in the DF, based on the updated protocol. This study aims to discuss the new recommendations for professionals in Primary Health Care (PHC) and maternity hospitals in DF, relating it to the case. This is a case report of a newborn exposed to syphilis in a public hospital in the DF in the sector of Joint Accommodation (ALCON) held in August 2020 during the mandatory practical activity of the uniprofessional residence in Obstetric Nursing. However, it was noted the importance of following the recommendations to minimize unnecessary damage and interventions for the family and newborn.
\end{abstract}

Key words: Congenital Syphilis. Treponema pallidum. Sexually transmitted diseases. 


\section{INTRODUÇÃO}

A Sífilis é uma Infecção Sexualmente Transmissível (IST) sistêmica, descoberta em 1905, causada por uma bactéria espiroqueta, o Treponema pallidum. Apresenta-se na forma adquirida, transmitida pelo contato sexual, e pela forma congênita por transmissão vertical para o feto por uma mãe com sífilis que não realizou o tratamento adequado ${ }^{1}$. A transmissão vertical acontece com mais frequência intra-útero, mas também pode ocorrer durante a passagem do feto pelo canal do parto, se houver lesão ativa. A sífilis na gestação pode resultar em aborto, parto prematuro, morte fetal e manifestação congênita precoce ou tardia ${ }^{2}$.

No Brasil, foi observado um aumento constante de casos nos últimos anos de sífilis em gestantes, sífilis congênita e sífilis adquirida, que são casos notificados pelo Sistema de Informação de Agravos de Notificação (SINAN) sendo obrigatória sua realização por profissionais de saúde ${ }^{3}$. Este aumento pode estar relacionado com a elevação de números de testagem, principalmente pelos testes rápidos que são de fácil acesso na Atenção Primária de Saúde (APS), mas também pela diminuição do uso de preservativos ${ }^{4}$.

Segundo o novo Boletim Epidemiológico, no Brasil, a sífilis adquirida teve sua taxa de detecção aumentada em 2017 de 59,1 casos por 100.000 habitantes, para 75,8 casos por 100.000 habitantes, em 2018. A taxa de detecção de sífilis em gestantes em 2018 foi de 21,4/1.000 nascidos vivos, a taxa de incidência de sífilis congênita foi de 9,0/1.000 nascidos vivos e taxa de mortalidade por sífilis congênita foi de $8,2 / 100.000$ nascidos vivos ${ }^{5}$. No Distrito Federal (DF), foram notificados 384 casos de sífilis congênita em 2018, apresentando 108 casos a mais em relação a 2017. O coeficiente de detecção apresenta crescimento desde 2013, sem desaceleração, com um aumento de 5,3\% entre 2013 e $2018^{6}$.

Portanto, considerando o impacto e o crescimento da Sífilis Congênita (SC), a Secretaria de Estado de Saúde do DF (SES-DF) emitiu a nota técnica No. 9/20 no mês de julho de 2020 com atualizações e orientações no manejo clínico e epidemiológico dos casos de SC no DF, visando melhorar a abordagem dos profissionais de saúde em todos os níveis de 
assistência, e este estudo tem como principal motivação, discutir a nova atualização sobre o tema.

\section{RELATO DE CASO}

Puérpera, 22 anos, G1Pn1A0, realizou pré-natal de risco habitual com sete consultas. Durante os exames do $1^{\circ}$ trimestre, o teste rápido para sífilis apresentou não reagente, porém, foi diagnosticada com sífilis gestacional após o resultado de o VDRL apresentar reagente com titulação de 1:64. A paciente refere tratamento com três doses de penicilina após descoberta, juntamente com o parceiro. No cartão da gestante, a medicação está prescrita na conduta do profissional, porém, não deixou explícito que foi realizado o tratamento adequado. A partir do $2^{\circ}$ trimestre de gestação, os resultados com o teste-rápido apresentaram positivos e queda progressiva na titulação materna, com resultado do VDRL de 1:32. Durante seu trabalho de parto, foi realizado um novo VDRL com titulação de 1:16.

A recém-nascida desta puérpera nasceu a termo, pesando $3,280 \mathrm{~kg}$, com apgar 09 no $1^{\circ}$ minuto e 10 no $5^{\circ}$ minuto. Ao exame físico não apresentava alterações clínicas. Durante a primeira avaliação pela equipe pediátrica, um dia após o parto, foi solicitado VDRL do recém-nascido que apresentou resultado 1:4. Devido ao não preenchimento correto do tratamento da gestante, foi solicitada radiografia de ossos longos e colhido líquor pela punção lombar, realizado no segundo dia de vida, e ambos com resultados negativos.

\section{DISCUSSÃO}

A sífilis é dividida em estágios baseados em achados clínicos que orientam o tratamento e o seguimento de cada caso. Em gestantes, a maioria dos casos é diagnosticado durante o pré-natal e o parto ${ }^{1}$.

Na sífilis adquirida, a forma mais comum de penetração do Treponema pallidum no organismo é por erosões cutâneas decorrentes da relação sexual. As espiroquetas penetram 
nas mucosas, aderem às células e se ligam aos receptores, proporcionando a fixação das espiroquetas, o que facilita a colonização da bactéria nos tecidos e órgãos. Como resultado da resposta imunológica localizada ocorre uma erosão e ulceração no ponto de inoculação, enquanto a disseminação sistêmica resulta no ataque imune circulante que pode depositar em qualquer órgão ${ }^{7}$.

Nos casos de SC, o T. pallidum é liberado diretamente na circulação fetal, podendo ocorrer em qualquer fase gestacional, porém é mais comum no primeiro trimestre da gestação no qual o fluxo placentário está mais ativo².

Durante uma infecção ocorre o reconhecimento dos lipopeptídeos do T. pallidum através das células dendríticas e os macrófagos, causam o estímulo da produção de citocinas inflamatórias que provocam uma resposta inflamatória severa ${ }^{7}$. O aumento da produção das citocinas inflamatórias, interleucina dois (IL-2), interferon gama (IFN-g), fator de necrose tumoral (TNF-a) e prostaglandinas induzidas por infecção uterina têm sido associados à morte fetal, retardo de crescimento e parto prematuro em qualquer fase da gestação ${ }^{7}$. Quando não ocorre morte fetal, resulta em ampla disseminação das espiroquetas por quase todos os órgãos e sistemas do feto. As manifestações clínicas decorrem da resposta inflamatória e são variáveis $^{7}$.

Na sífilis adquirida, as manifestações clínicas são de acordo com a evolução dos estágios. Na Sífilis recente (menos de 2 anos de duração) temos três estágios: primária (10-90 dias, média de 3 semanas após o contato); secundária (6 semanas a 6 meses após o contato); e latente recente (nos primeiros 2 anos da infecção). A fase primária se manifesta com um nódulo único e se ulcera formando o cancro duro. $\mathrm{Na}$ fase secundária são comuns sinais e sintomas sistêmicos, podendo ocorrer erupções cutâneas em forma de máculas e/ou pápulas. Na sífilis tardia (mais de 2 anos de duração), temos o estágio latente tardia (após 2 anos de infecção) e a terciária. Nos estágios de latente recente e tardia, são períodos em que não se 
observa sinal ou sintoma clinico de sífilis, são diagnosticados somente com testes imunológicos. A terciária é menos frequente, acomete o sistema nervoso e cardiovascular ${ }^{2}$.

A avaliação inicial da criança exposta à sífilis ou com SC é realizada na maternidade considerando o histórico materno de sífilis quanto ao tratamento e seguimento na gestação, sinais e sintomas clínicos da criança, teste não treponêmico periférico do recém-nascido comparado com o da mãe. O diagnóstico é estabelecido pela combinação da avaliação clínica, epidemiológica e laboratorial ${ }^{2,6}$.

A SC precoce pode surgir até o segundo ano de vida. Já a SC tardia é definida como aquela em que os sinais e sintomas surgem após os dois anos de idade da criança. Sendo necessária atenção específica aos sinais e sintomas mais clássicos, referentes às manifestações ${ }^{2}$.

As manifestações clínicas da SC precoce são inespecíficas e podem ser encontradas em outras infecções congênitas, porém, possui alterações mais comuns, como a hepatomegalia, atraso no desenvolvimento neuropsicomotor, rinite sifilítica ou corrimento nasal, anemia, trombocitopenia, leucopenia, leucocitose, anormalidades radiográficas, anormalidades no líquido cefalorraquidiano, entre outras. Já as manifestações clínicas da SC tardia estão relacionadas à inflamação cicatricial ou persistente da infecção precoce e se caracterizam pela presença de formação das gomas sifilíticas em diversos tecidos, as manifestações mais comuns são na face (fronte olímpica e nariz em sela), alterações oftalmológicas (coriorretinite, glaucoma secundário e atrofia óptica), auditivas (perda auditiva sensorial), orofaríngeas (perfuração do palato duro), sistema nervoso central (atraso no desenvolvimento, hidrocefalia, crises convulsivas, atrofia do nervo óptico) esquelético (tíbia em sabre), entre outras ${ }^{2,6}$.

Os testes para o diagnóstico da sífilis são divididos em duas categorias: exames diretos e testes imunológicos. Nos exames diretos, é visualizada a presença das espiroquetas no 
material retirado das lesões primárias ou secundárias ativas. Os testes imunológicos são os mais utilizados, dividem-se em treponêmicos e não treponêmicos ${ }^{2}$. Os treponêmicos detectam anticorpos específicos produzidos contra os antígenos do Treponema pallidum, são os primeiros a se tornarem reagentes e permanecem mesmo após o tratamento, não são indicados para monitoramento da resposta ao tratamento (teste rápido, FTA-Abs, ELISA/EQL, TPHA/TPPA/MHA-TP) ${ }^{2}$. Os testes não treponêmicos detectam anticorpos não específicos anticardiolipina, material lipídico liberado pelas células danificadas e possivelmente contra a cardiolipina liberada pelos treponemas, são quantificáveis e devem ter seu resultado em frações para melhor diagnóstico e monitoramento da resposta ao tratamento (VDRL, RPR, TRUST) ${ }^{2}$. O VDRL é o exame mais utilizado para rastreamento durante o pré-natal, o teste torna-se positivo cinco a seis semanas após a infecção, motivo pelo qual, não se encontra positivo na fase de manifestação clínica do cancro. Esse exame é titulado, ou seja, diluído, e apresenta títulos elevados quando há infecção aguda e, principalmente, na fase secundária da doença ${ }^{8}$.

Nas orientações da Secretaria de Estado de Saúde do Distrito Federal (SES-DF) para os profissionais de saúde da atenção secundária e maternidades quanto ao risco da SC, é recomendado que essas crianças sejam investigadas ainda na maternidade quanto às manifestações clínicas e exames complementares ${ }^{6}$. Todos os recém-nascidos de mãe com diagnóstico de sífilis na gestação devem realizar teste não treponêmico periférico ${ }^{6}$. O sangue de cordão umbilical não deve ser utilizado, pois esse tipo de amostra contém uma mistura do sangue da criança com o materno e pela contaminação da geleia de Wharton que pode resultar em testes falso-positivo ${ }^{2}$.

As crianças são classificadas como caso de SC quando a mãe não foi tratada ou não realizou o tratamento adequado durante o pré-natal, independente dos resultados clínicos ou exames $^{6}$. E o outro caso, independente do histórico de tratamento da mãe, as crianças com 
resultado de teste não treponêmicos maior que o da mãe em pelo menos duas diluições, devendo ser notificadas, investigadas tratadas e acompanhadas. Todas as crianças com SC devem ser submetidas a uma investigação completa, incluindo radiografia de ossos longos, hemograma, punção lombar e análise do líquor ${ }^{6}$.

A criança exposta à sífilis é aquela nascida assintomática, cuja mãe foi adequadamente tratada e cujo teste não treponêmico é não reagente ou reagente com titulação menor, igual ou até uma diluição maior que o materno ${ }^{2}$. É importante identificar adequadamente crianças expostas (mas não infectadas) para não realizar condutas desnecessárias, como exames invasivos e internações prolongadas ${ }^{2,6}$. É essencial garantir o seguimento de todas as crianças expostas à sífilis, na perspectiva de que elas podem desenvolver sinais e sintomas mais tardios, independentemente da primeira avaliação e/ou tratamento na maternidade ${ }^{6}$.

Durante a gestação, a testagem para sífilis no DF está preconizada na $1^{\text {a }}$ consulta de pré-natal, idealmente no $1^{\circ}, 2^{\circ}$ e $3^{\circ}$ trimestre, no momento do parto, em casos de aborto e exposição de risco ou violência sexual ${ }^{1}$.

Nas novas recomendações aos profissionais de saúde do pré-natal, destaca que em gestantes, o tratamento deve ser iniciado com apenas um teste reagente, sem aguardar o resultado do segundo teste devido ao tempo de exposição e risco de infecção para o feto ${ }^{6}$. A benzilpenicilina benzatina é segura e a melhor opção para o tratamento, o profissional deve garantir o tratamento adequado e registrá-lo na caderneta da gestante, para impedir que o recém-nascido passe por intervenções desnecessárias ${ }^{6}$. Os parceiros sexuais de gestantes com sífilis devem ser tratados, o não tratamento pode levar a reinfecção das gestantes. Todos os casos de crianças expostas à sífilis ou SC até 18 ou 24 meses de vida, devem ser encaminhadas para seguimento na APS para melhor monitoramento, além da realização de exames complementares ${ }^{6}$. 
A SC pode trazer complicações para a criança, os casos devem ser avaliados no seguimento pós-neonatal, a cada seis meses, durante dois anos ${ }^{6}$. As avaliações são necessárias para detectar alterações oftalmológicas, audiológicas e neurológicas. Para casos com alterações liquóricas na maternidade é recomendada a coleta de líquor a cada 6 meses, até a sua normalização. Estas avaliações serão encaminhadas pela APS ${ }^{6}$.

Outras recomendações da nova nota técnica incluem, que crianças nascidas de mulheres com diagnóstico de sífilis anterior a gestação atual, com histórico documentado de tratamento, queda da titulação e que durante a gestação atual se mantiveram com títulos de teste não treponêmico baixos e estáveis, não são consideradas crianças expostas à sífilis, e não precisam coletar VDRL no momento do parto $^{6}$. Deve ter o seguimento ambulatorial clínico e com testes não treponêmicos por 18 a 24 meses para todas as crianças com SC ou expostas à sífilis. Suspender a realização de teste não treponêmico quando obtiver dois resultados não reagentes consecutivos, durante o seguimento ambulatorial da criança. Realizar avaliação audiológica, neurológica e oftalmológica, a cada seis meses durante dois anos. Realizar teste treponêmico após os 18 meses de idade ${ }^{6}$.

O esquema terapêutico da gestante com sífilis é classificada segundo seu estágio clinico. A sífilis recente deve ser tratada com Penicilina $G$ benzatina 2,4 milhões UI por via intramuscular (IM) em dose única. Na sífilis tardia deve ser tratada com Penicilina G benzatina 2,4 milhões UI, IM, semanal, por 3 semanas seguidas, e dose total de 7,2 milhões $\mathrm{UI}^{6}$. Os parceiros sexuais das gestantes podem estar infectados, mesmo apresentando testes imunológicos não reagentes e devem ser tratados com apenas uma dose de penicilina benzatina 2,4 milhões UI, IM, em casos de sífilis recente. Em sífilis tardia o tratamento deve ser por 3 semanas igualmente o da gestante ${ }^{2,6}$.

Para tratamento de crianças com sífilis congênita é utilizado a benzilpenicilina, a depender do tratamento materno durante a gestação e titulação da criança comparada ao 
materno ou exames clínicos e laboratoriais da criança. A única situação em que não é necessário tratamento é a da criança exposta à sífilis² .

Para os recém-nascidos em que a mãe não foi tratada adequadamente e apresentou exame físico, hemograma, radiografia, líquor normais e o teste não treponêmico não reagente, deve-se aplicar uma dose IM de benzilpenicilina benzatina $50.000 \mathrm{UI} / \mathrm{kg}$ para o tratamento profilático $^{2}$. Já a criança com SC e alteração nos exames deve passar pelo tratamento de 10 dias na maternidade, a depender do caso ${ }^{6}$.

\section{CONCLUSÕES}

Nos últimos anos, houve um crescimento significativo nos casos de sífilis em gestantes e SC, principalmente pelo aumento no número de testagens, o não uso de preservativos e por esses casos entrarem na lista de notificação compulsória a partir de 2017, sendo comum o diagnóstico e tratamento dessas gestantes durante o pré-natal e o seguimento dessas crianças na maternidade e pela APS.

Desta forma, o presente relato de caso buscou alertar a importância de seguir o protocolo atualizado pelos profissionais de saúde que participam de toda assistência envolvida nos casos de sífilis em gestante e SC, para minimizar danos e intervenções desnecessárias para as famílias, principalmente ao recém-nascido na maternidade que está mais sujeito a procedimentos invasivos. Nesse sentido, a nota técnica da SES-DF traz recomendações para que exista um padrão de atendimento que deve ser seguido pelos profissionais desta secretaria.

\section{REFERÊNCIAS}

1. Farias, C., Medeiros, J. Ocorrência de sífilis em gestantes nas macrorregiões de saúde do estado da Paraíba, Brasil, de 2014 a 2018. Journal of Biology \& Pharmacy. Vol. 15 n4. 
http://revista.uepb.edu.br/index.php/biofarm/article/view/5291/3073

2. Brasil. Ministério da Saúde. Secretaria de Vigilância em Saúde. Departamento de Doenças de Condições Crônicas e Infecções Sexualmente Transmissíveis. Protocolo Clínico e Diretrizes Terapêuticas para Prevenção da Transmissão Vertical do HIV, Sífilis e Hepatites Virais - Brasília: Ministério da Saúde, 2019. 248 p. Disponível em: http://www.aids.gov.br/pt-br/pub/2015/protocolo-clinico-e-diretrizes-terapeuticas-paraprevencao-da-transmissao-vertical-de-hiv

3. Lafetá, Kátia Regina Gandra et al. Sífilis materna e congênita, subnotificação e difícil controle. Revista Brasileira de Epidemiologia [online]. 2016, v. 19, n. 01, pp. 63-74. Disponível em: https://www.scielo.br/pdf/rbepid/v19n1/1980-5497-rbepid-19-01-00063.pdf

4. Brasil. Portaria de consolidação $n^{\circ}$ 4/2017. Ministério da Saúde, Brasília, 28 de setembro de 2017. Disponível em: http://bvsms.saude.gov.br/bvs/saudelegis/gm/2017/prc0004_03_10_2017.html 5. Brasil. Ministério da Saúde. Secretaria de Vigilância em Saúde. Boletim Epidemiológico. Número Especial | Out. 2019. Disponível em: https://portaldeboaspraticas.iff.fiocruz.br/biblioteca/boletim-epidemiologico-sifilis-2019/

6. Governo do Distrito Federal. Secretaria de Estado de Saúde do Distrito Federal. Diretoria de Vigilância Epidemiológica. Gerência de Vigilância de Infecções Sexualmente Transmissíveis. Nota Técnica N. ${ }^{\circ}$ 9/2020. Julho, 2020.

7. Silva, Gláucia Cristina Barbosa. Rodrigues, Fernando Fachinelli. Fisiopatologia da sífilis congênita. Revista Científica Multidisciplinar Núcleo do Conhecimento. Ano 03, Ed. 10, Vol. 04, pp. 122-136. Outubro de 2018. Disponível em: https://www.nucleodoconhecimento.com.br/saude/fisiopatologia

8. Zugaib obstetrícia. Editor: Marcelo Zugaib. - 3. ed. Barueri, SP: Manole, 2016. 